\title{
Refinements of Hermite-Hadamard type inequalities for operator convex functions
}

\section{Vildan Bacak ${ }^{*}$ and Ramazan Türkmen}

\section{"Correspondence:}

vildanbacak@selcuk.edu.tr

Department of Mathematics,

Science Faculty, Selçuk University,

Konya, Turkey

\begin{abstract}
The purpose of this paper is to present some new versions of Hermite-Hadamard type inequalities for operator convex functions. We give refinements of Hermite-Hadamard type inequalities for convex functions of self-adjoint operators in a Hilbert space analogous to well-known inequalities of the same type. The results presented in this paper are more general than known results given by several authors.
\end{abstract} MSC: 26D15; 47A63

Keywords: Hermite-Hadamard inequality; operator convex functions; self-adjoint operators

\section{Introduction}

Let $f$ be a real-valued function defined on $I \in \mathbb{R}$. The function $f$ is called convex if

$$
f(\lambda a+(1-\lambda) b) \leq \lambda f(a)+(1-\lambda) f(b)
$$

for all $\lambda \in[0,1]$ and $a, b \in I$. The function $f$ is called concave if

$$
f(\lambda a+(1-\lambda) b) \geq \lambda f(a)+(1-\lambda) f(b)
$$

for all $\lambda \in[0,1]$ and $a, b \in I$. Let $f:[a, b] \rightarrow \mathbb{R}$ be a convex function and $a, b \in \mathbb{R}$, with $a<b$, then the inequality

$$
f\left(\frac{a+b}{2}\right) \leq \frac{1}{b-a} \int_{a}^{b} f(x) d x \leq \frac{f(a)+f(b)}{2}, \quad a, b \in \mathbb{R},
$$

is known in the literature as the Hermite-Hadamard inequality for convex functions, see [1]. Such inequality is very useful in many mathematical contexts and contributes as a tool for establishing some interesting estimations. Both inequalities in (1.1) hold in the reversed direction if $f$ is concave.

Let $X$ be a vector space, $x, y \in X, x \neq y$ and $[x, y]=\{(1-t) x+t y, t \in[0,1]\}$. We consider the function $f:[x, y] \rightarrow \mathbb{R}$ and the associated function

$$
g(x, y):[0,1] \rightarrow \mathbb{R}, \quad g(x, y)(t):=f[(1-t) x+t y], \quad t \in[0,1] .
$$

Note that $f$ is convex on $[x, y]$ if and only if $g(x, y)$ is convex on $[0,1]$.

\section{Springer}

C) 2013 Bacak and Türkmen; licensee Springer. This is an Open Access article distributed under the terms of the Creative Commons Attribution License (http://creativecommons.org/licenses/by/2.0), which permits unrestricted use, distribution, and reproduction in any medium, provided the original work is properly cited. 
For any convex function $f$ defined on a segment $[x, y] \subset X$, we have the HermiteHadamard integral inequality

$$
f\left(\frac{x+y}{2}\right) \leq \int_{0}^{1} f[(1-t) x+t y] d t \leq \frac{f(x)+f(y)}{2},
$$

which can be derived from the classical Hermite-Hadamard inequality (1.1) for the convex function $g(x, y):[0,1] \rightarrow \mathbb{R}$.

On a finite-dimensional inner product space, a self-adjoint operator is an operator that is its own adjoint, or, equivalently, one whose matrix is Hermitian, where a Hermitian matrix is one which is equal to its own conjugate transpose.

A real-valued continuous function $f$ on an interval $I$ is said to be operator convex (operator concave) if

$$
f((1-\lambda) A+\lambda B) \leq(\geq)(1-\lambda) f(A)+\lambda f(B)
$$

in the operator order for all $\lambda \in[0,1]$ and for every self-adjoint operator $A$ and $B$ on a Hilbert space $H$ whose spectra are contained in $I$. Notice that a function $f$ is operator concave if $-f$ is operator convex.

In recent years many authors have been interested in giving some refinements and extensions of the Hermite-Hadamard inequality (1.1). For more about convex functions and the Hermite-Hadamard inequality, see [2-6].

The author in [7] presents the Hermite-Hadamard type inequality for convex functions by sequences. But the inequality therein is established on $2^{n}$. In this paper, a new refinement of the Hermite-Hadamard type inequality is presented. Our inequality is an improved version of the inequality given in [7]. Namely, this inequality includes not only $2^{n}$, but also all positive real numbers as the number of partition.

The author in [8] shows some new integral inequalities analogous to the well-known Hermite-Hadamard inequality. We give a general form of the first of these inequalities and show that the inequalities therein are satisfied for operator convex functions.

View more results about operator convex functions and Hermite-Hadamard type inequalities in [9]. The authors in [9] show further results analogous to the results in this paper.

Dragomir proved the following theorem in [3].

Theorem 1 Let $f: I \rightarrow \mathbb{R}$ be an operator convex function on some interval $I$. Then, for any self-adjoint operators $A$ and $B$ with spectra in I, we have the inequality

$$
\begin{aligned}
& \left(f\left(\frac{A+B}{2}\right) \leq\right) \frac{1}{2}\left[f\left(\frac{3 A+B}{4}\right)+f\left(\frac{A+3 B}{4}\right)\right] \\
& \quad \leq \int_{0}^{1} f((1-t) A+t B) d t \\
& \quad \leq \frac{1}{2}\left[f\left(\frac{A+B}{2}\right)+\frac{f(A)+f(B)}{2}\right]\left(\leq \frac{f(A)+f(B)}{2}\right) .
\end{aligned}
$$

Zabandan gave a refinement of the Hermite-Hadamard inequality for convex functions in [7]. 
Theorem 2 Let $f$ be a convex function on $[a, b]$. Then we have

$$
\begin{aligned}
f\left(\frac{a+b}{2}\right) & =x_{0} \leq \frac{1}{2}\left[f\left(\frac{3 a+b}{4}\right)+f\left(\frac{a+3 b}{4}\right)\right] \\
& =x_{1} \leq \cdots \leq x_{n} \leq \cdots \leq \frac{1}{b-a} \int_{a}^{b} f(x) d x \leq \cdots \leq y_{n} \\
& \leq \cdots \leq y_{1}=\frac{1}{4}\left[f(a)+2 f\left(\frac{a+b}{2}\right)+f(b)\right] \\
& \leq y_{0}=\frac{f(a)+f(b)}{2},
\end{aligned}
$$

where

$$
x_{n}=\frac{1}{2^{n}} \sum_{i=1}^{2^{n}} f\left(a+i \frac{b-a}{2^{n}}-\frac{b-a}{2^{n+1}}\right)=\frac{1}{2^{n}} \sum_{i=1}^{2^{n}} f\left(a+\left(i-\frac{1}{2}\right) \frac{b-a}{2^{n}}\right)
$$

and

$$
\begin{aligned}
y_{n} & =\frac{1}{2^{n+1}} \sum_{i=1}^{2^{n}} f\left(\left(1-\frac{i}{2^{n}}\right) a+\frac{i}{2^{n}} b\right)+f\left(\left(1-\frac{i-1}{2^{n}}\right) a+\frac{i-1}{2^{n}} b\right) \\
& =\frac{1}{2^{n+1}}\left[f(a)+f(b)+2 \sum_{i=1}^{2^{n}-1} f\left(\left(1-\frac{i}{2^{n}}\right) a+\frac{i}{2^{n}} b\right)\right] .
\end{aligned}
$$

Pachpatte gave some integral inequalities analogous to the well-known HermiteHadamard inequality by using a fairly elementary analysis in [8] as follows.

Theorem 3 Let $f$ and $g$ be real-valued, nonnegative and convex functions on $[a, b]$. Then

(i) $\frac{1}{b-a} \int_{a}^{b} f(x) g(x) d x \leq \frac{1}{3} M(a, b)+\frac{1}{6} N(a, b)$,

(ii) $2 f\left(\frac{a+b}{2}\right) g\left(\frac{a+b}{2}\right) \leq \frac{1}{b-a} \int_{a}^{b} f(x) g(x) d x+\frac{1}{6} M(a, b)+\frac{1}{3} N(a, b)$,

where $M(a, b)=f(a) g(a)+f(b) g(b), N(a, b)=f(a) g(b)+f(b) g(a)$.

\section{Main results}

Theorem 4 Let $f: I \rightarrow \mathbb{R}$ be an operator convex function on some interval $I$. Then for any self-adjoint operators $A$ and $B$ with spectra in $I$, we have the inequality

$$
\begin{aligned}
& \left(f\left(\frac{A+B}{2}\right) \leq\right) \frac{1}{k} \sum_{i=0}^{k-1} f\left(\frac{(2 k-2 i-1) A+(2 i+1) B}{2 k}\right) \\
& \quad \leq \int_{0}^{1} f((1-t) A+t B) d t \\
& \quad \leq \frac{1}{k}\left[\sum_{i=1}^{k-1} f\left(\frac{(k-i) A+i B}{k}\right)+\frac{f(A)+f(B)}{2}\right]\left(\leq \frac{f(A)+f(B)}{2}\right),
\end{aligned}
$$

where $k$ is the number of steps. 
Proof The function $f$ is continuous, $\int_{0}^{1} f((1-t) A+t B) d t$ exists for any self-adjoint operators $A$ and $B$ with spectra in $I$.

We can give two proofs of the theorem. The first using the definition of operator convex functions and the second using the Hermite-Hadamard inequality for real-valued functions.

1. From the definition of operator convex functions, we have the inequalities

$$
\begin{aligned}
f\left(\frac{X+Y}{2}\right) & =f\left(\frac{(1-t) X+t Y}{2}+\frac{(1-t) Y+t X}{2}\right) \\
& \leq \frac{f((1-t) X+t Y)+f((1-t) Y+t X)}{2} \\
& \leq \frac{f(X)+f(Y)}{2}
\end{aligned}
$$

for any $t \in[0,1]$ and self-adjoint operators $X$ and $Y$ with spectra in $I$. If we integrate the inequality (2.2) over $t$ and take into account that

$$
\int_{0}^{1} f((1-t) X+t Y) d t=\int_{0}^{1} f(t X+(1-t) Y) d t
$$

then we conclude the Hermite-Hadamard inequality for operator convex functions

$$
\begin{aligned}
f\left(\frac{X+Y}{2}\right) & \leq \int_{0}^{1} f((1-t) X+t Y) d t \\
& \leq \frac{f(X)+f(Y)}{2}
\end{aligned}
$$

that holds for any self-adjoint operators $X$ and $Y$ with spectra in $I$. Utilizing the change of variable $u=k t$, we have

$$
\begin{aligned}
\int_{0}^{\frac{1}{k}} f((1-t) A+t B) d t & =\frac{1}{k} \int_{0}^{1} f\left(\left(1-\frac{u}{k}\right) A+\frac{u}{k} B\right) d u \\
& =\frac{1}{k} \int_{0}^{1} f\left(A-\frac{A u}{k}+\frac{B u}{k}\right) d u \\
& =\frac{1}{k} \int_{0}^{1} f\left((1-u) A+u \frac{(k-1) A+B}{k}\right) d u
\end{aligned}
$$

and by the change of variable $u=k t-1$, we have

$$
\begin{aligned}
\int_{\frac{1}{k}}^{\frac{2}{k}} f((1-t) A+t B) d t & =\frac{1}{k} \int_{0}^{1} f\left(\left(1-\frac{u+1}{k}\right) A+\frac{u+1}{k} B\right) d u \\
& =\frac{1}{k} \int_{0}^{1} f\left(A-\frac{A u}{k}-\frac{A}{k}+\frac{B u}{k}+\frac{B}{k}\right) d u \\
& =\frac{1}{k} \int_{0}^{1} f\left((1-u) \frac{(k-1) A+B}{k}+u \frac{(k-2) A+2 B}{k}\right) d u .
\end{aligned}
$$


We can change the variables until the variable $u=k t-(k-1)$ by using the same procedure above. By the change of variable $u=k t-(k-1)$, we get

$$
\begin{aligned}
\int_{\frac{k-1}{k}}^{1} f((1-t) A+t B) d t & =\frac{1}{k} \int_{0}^{1} f\left(\left(1-\frac{u+k-1}{k}\right) A+\frac{u+k-1}{k} B\right) d u \\
& =\frac{1}{k} \int_{0}^{1} f\left(A-\frac{A u}{k}-A+\frac{A}{k}+\frac{B u}{k}+B-\frac{B}{k}\right) d u \\
& =\frac{1}{k} \int_{0}^{1} f\left((1-u) \frac{A+(k-1) B}{k}+u B\right) d u .
\end{aligned}
$$

Using the Hermite-Hadamard inequality in (2.3), we have

$$
\begin{aligned}
& f\left(\frac{A+\frac{(k-1) A+B}{k}}{2}\right)=f\left(\frac{(2 k-1) A+B}{2 k}\right) \\
& \leq \int_{0}^{1} f\left((1-u) A+u \frac{(k-1) A+B}{k}\right) d u \\
& \leq \frac{1}{2}\left[f(A)+f\left(\frac{(k-1) A+B}{k}\right)\right] \text {, } \\
& f\left(\frac{\frac{(k-1) A+B}{k}+\frac{(k-2) A+2 B}{k}}{2}\right)=f\left(\frac{(2 k-3) A+3 B}{2 k}\right) \\
& \leq \int_{0}^{1} f\left((1-u) \frac{(k-1) A+B}{k}+u \frac{(k-2) A+2 B}{k}\right) d u \\
& \leq \frac{1}{2}\left[f\left(\frac{(k-1) A+B}{k}\right)+f\left(\frac{(k-2) A+2 B}{k}\right)\right], \\
& f\left(\frac{\frac{(k-2) A+2 B}{k}+\frac{(k-3) A+3 B}{k}}{2}\right) \\
& =f\left(\frac{(2 k-5) A+5 B}{2 k}\right) \\
& \leq \int_{0}^{1} f\left((1-u) \frac{(k-2) A+2 B}{k}+u \frac{(k-3) A+3 B}{k}\right) d u \\
& \leq \frac{1}{2}\left[f\left(\frac{(k-2) A+2 B}{k}\right)+f\left(\frac{(k-3) A+3 B}{k}\right)\right] \text {, }
\end{aligned}
$$

By induction we have

$$
\begin{aligned}
f\left(\frac{\frac{A+(k-1) B}{k}+B}{2}\right) & =f\left(\frac{A+(2 k-1) B}{2 k}\right) \\
& \leq \int_{0}^{1} f\left((1-u) \frac{A+(k-1) B}{k}+u B\right) d u \\
& \leq \frac{1}{2}\left[f\left(\frac{A+(k-1) B}{k}\right)+f(B)\right] .
\end{aligned}
$$


By summing (2.4), (2.5), (2.6), (2.7) and the other inequalities between (2.6) and (2.7), we have

$$
\begin{aligned}
f( & \left.\frac{A+\frac{(k-1) A+B}{k}}{2}\right)+f\left(\frac{\frac{(k-1) A+B}{k}+\frac{(k-2) A+2 B}{k}}{2}\right) \\
& +f\left(\frac{\frac{(k-2) A+2 B}{k}+\frac{(k-3) A+3 B}{k}}{2}\right)+\cdots+f\left(\frac{\frac{A+(k-1) B}{k}+B}{2}\right) \\
\leq & k \int_{0}^{1} f((1-t) A+t B) d t \\
\leq & \frac{1}{2}\left[f(A)+2 f\left(\frac{(k-1) A+B}{k}\right)+2 f\left(\frac{(k-2) A+2 B}{k}\right)+\cdots\right. \\
& \left.+2 f\left(\frac{A+(k-1) B}{k}\right)+f(B)\right] .
\end{aligned}
$$

When regulating the inequality (2.8), we get the desired inequality in (2.1). It is obvious from the left-hand side of the inequality (2.1) for $k=1$, we get $f\left(\frac{A+B}{2}\right)$, and it is obvious the right-hand side of the inequality (2.1) is provided for $k=2$.

2. Let $x \in H,\|x\|=1$ and let $A$ and $B$ be two self-adjoint operators with spectra in $I$. Define the real-valued function $\varphi_{x, A, B}:[0,1] \rightarrow \mathbb{R}$ by $\varphi_{x, A, B}(t)=\langle f((1-t) A+t B) x, x\rangle$. Since $f$ is operator convex, then for any $t_{1}, t_{2} \in[0,1]$ and $\alpha, \beta \geq 0$ with $\alpha+\beta=1$, we have

$$
\begin{aligned}
\varphi_{x, A, B}\left(\alpha t_{1}+\beta t_{2}\right)= & \left\langle f\left(\left(1-\left(\alpha t_{1}+\beta t_{2}\right)\right) A+\left(\alpha t_{1}+\beta t_{2}\right) B\right) x, x\right\rangle \\
= & \left\langle f\left(\alpha\left[\left(1-t_{1}\right) A+t_{1} B\right]+\beta\left[\left(1-t_{2}\right) A+t_{2} B\right]\right) x, x\right\rangle \\
\leq & \alpha\left\langle f\left(\left[\left(1-t_{1}\right) A+t_{1} B\right]\right) x, x\right\rangle \\
& +\beta\left\{f\left(\beta\left[\left(1-t_{2}\right) A+t_{2} B\right]\right) x, x\right\rangle \\
= & \alpha \varphi_{x, A, B}\left(t_{1}\right)+\beta \varphi_{x, A, B}\left(t_{2}\right)
\end{aligned}
$$

showing that $\varphi_{x, A, B}$ is a convex function on $[0,1]$. Now we can use the Hermite-Hadamard inequality for real-valued functions

$$
g\left(\frac{a+b}{2}\right) \leq \frac{1}{b-a} \int_{a}^{b} g(s) d s \leq \frac{g(a)+g(b)}{2}
$$

to get that

$$
\begin{aligned}
& \varphi_{x, A, B}\left(\frac{1}{2 k}\right) \leq k \int_{0}^{\frac{1}{k}} \varphi_{x, A, B}(t) d t \leq \frac{\varphi_{x, A, B}(0)+\varphi_{x, A, B}(1 / k)}{2}, \\
& \varphi_{x, A, B}\left(\frac{3}{2 k}\right) \leq k \int_{\frac{1}{k}}^{\frac{2}{k}} \varphi_{x, A, B}(t) d t \leq \frac{\varphi_{x, A, B}\left(\frac{1}{k}\right)+\varphi_{x, A, B}\left(\frac{2}{k}\right)}{2}, \\
& \vdots \\
& \varphi_{x, A, B}\left(\frac{2 k-1}{2 k}\right) \leq k \int_{\frac{k-1}{k}}^{1} \varphi_{x, A, B}(t) d t \leq \frac{\varphi_{x, A, B}\left(\frac{k-1}{k}\right)+\varphi_{x, A, B}(1)}{2} .
\end{aligned}
$$


By summing the inequalities above and multiplying with $\frac{1}{k}$, we get

$$
\begin{aligned}
\frac{1}{k} & {\left[\varphi_{x, A, B}\left(\frac{1}{2 k}\right)+\varphi_{x, A, B}\left(\frac{3}{2 k}\right)+\cdots+\varphi_{x, A, B}\left(\frac{2 k-1}{2 k}\right)\right] } \\
& \leq \int_{0}^{1} \varphi_{x, A, B}(t) d t \\
& \leq \frac{1}{k}\left[\frac{\varphi_{x, A, B}(0)+\varphi_{x, A, B}(1)}{2}+\varphi_{x, A, B}\left(\frac{1}{k}\right)+\varphi_{x, A, B}\left(\frac{2}{k}\right)+\cdots+\varphi_{x, A, B}\left(\frac{k-1}{k}\right)\right] .
\end{aligned}
$$

Thus, we can write

$$
\begin{aligned}
& \frac{1}{k}\left\langle\left[ f\left(\left(1-\frac{2}{k}\right) A+\frac{1}{2 k} B\right)+f\left(\left(1-\frac{3}{2 k}\right) A+\frac{3}{2 k} B\right)+\cdots\right.\right. \\
& \left.\left.\quad+f\left(\left(1-\frac{2 k-1}{2 k}\right) A+\frac{2 k-1}{2 k} B\right)\right] x, x\right\rangle \\
& \leq \int_{0}^{1}\langle f((1-t) A+t B) x, x\rangle d t \\
& \leq \frac{1}{k}\left\langle\left[\frac{f(A)+f(B)}{2}+f\left(\left(1-\frac{1}{k}\right) A+\frac{1}{k} B\right)+f\left(\left(1-\frac{2}{k}\right) A+\frac{2}{k} B\right)+\cdots\right.\right. \\
& \left.\left.\quad+f\left(\left(1-\frac{k-1}{k}\right) A+\frac{k-1}{k} B\right)\right] x, x\right\rangle .
\end{aligned}
$$

By regulating these inequalities above, we get

$$
\begin{aligned}
& \frac{1}{k}\left\langle\left[\sum_{i=0}^{k-1} f\left(\frac{(2 k-2 i-1) A+(2 i+1) B}{2 k}\right)\right] x, x\right\rangle \\
& \quad \leq \int_{0}^{1}\langle f((1-t) A+t B) x, x\rangle d t \\
& \quad \leq \frac{1}{k}\left\langle\left[\frac{f(A)+f(B)}{2}+\sum_{i=0}^{k-1} f\left(\frac{(k-i) A+i B}{k}\right)\right] x, x\right\rangle .
\end{aligned}
$$

Finally, since by the continuity of the function $f$, we have

$$
\int_{0}^{1}\langle f((1-t) A+t B) x, x\rangle d t=\left\langle\int_{0}^{1} f((1-t) A+t B) d t x, x\right\rangle
$$

for any $x \in H$, and any two self-adjoint operators $A$ and $B$ with spectra in $I$, from (2.9) we get the desired result in (2.1).

Remark 5 Our result for operator convex functions in Theorem 4 is more general than the inequality in Theorem 1. In the inequality (2.1) if we take $k=2$, we get the inequality in (1.3).

Remark 6 Our result for operator convex functions in Theorem 4 is more general than the inequality in Theorem 2. In the inequality (2.1), if we take $k=2^{n}$, we get the inequality in (1.4). In Theorem 2 , there are no cases of $k \in \mathbb{N} \backslash\left\{2^{n}, n=0,1,2, \ldots\right\}$. But our result involves these statements. 
Theorem 7 Let $f, g: I \rightarrow \mathbb{R}$ be an operator convex function on some interval I. Then for any self-adjoint operators $A$ and $B$ with spectra in $I$, we have the inequality

$$
\begin{aligned}
& \left.\int_{0}^{1}\langle f((1-t) A+t B) x, x) / g((1-t) A+t B) x, x\right\rangle d t \\
& \quad \leq \frac{1}{3} M(A, B)+\frac{1}{6} N(A, B),
\end{aligned}
$$

where

$$
\begin{aligned}
& M(A, B)=\langle f(A) x, x\rangle\langle g(A) x, x\rangle+\langle f(B) x, x\rangle\langle g(B) x, x\rangle, \\
& N(A, B)=\langle f(A) x, x\rangle\langle g(B) x, x\rangle+\langle f(B) x, x\rangle\langle g(A) x, x\rangle .
\end{aligned}
$$

Proof Let $x \in H,\|x\|=1$ and let $A$ and $B$ be two self-adjoint operators with spectra in $I$. Define the real-valued functions $\varphi_{x, A, B}:[0,1] \rightarrow \mathbb{R}$ by $\varphi_{x, A, B}(t)=\langle f((1-t) A+t B) x, x\rangle$ and $\psi_{x, A, B}:[0,1] \rightarrow \mathbb{R}$ by $\psi_{x, A, B}(t)=\langle g((1-t) A+t B) x, x\rangle$. Since $f$ and $g$ are operator convex functions, then for every $t \in[0,1]$, we have

$$
\begin{aligned}
& \langle f((1-t) A+t B) x, x\rangle \leq(1-t)\langle f(A) x, x\rangle+t|f(B) x, x\rangle, \\
& \langle g((1-t) A+t B) x, x\rangle \leq(1-t)\langle g(A) x, x\rangle+t|g(B) x, x\rangle .
\end{aligned}
$$

From (2.11) and (2.12), we obtain

$$
\begin{aligned}
& \langle f((1-t) A+t B) x, x)\langle g((1-t) A+t B) x, x\rangle \\
& \quad \leq(1-t)^{2}\langle f(A) x, x)\langle g(A) x, x\rangle+t^{2}\langle f(B) x, x\rangle\langle g(B) x, x\rangle \\
& \quad+t(1-t)(\langle f(A) x, x)\langle g(B) x, x\rangle+\langle f(B) x, x\rangle\langle g(A) x, x\rangle) .
\end{aligned}
$$

Since $\varphi_{x, A, B}(t)$ and $\psi_{x, A, B}(t)$ are operator convex on $[0,1]$, they are integrable on $[0,1]$ and consequently $\varphi_{x, A, B}(t) \psi_{x, A, B}(t)$ is also integrable on $[0,1]$. Integrating both sides of the inequality (2.13) over $[0,1]$, we get

$$
\begin{aligned}
& \int_{0}^{1}\langle f((1-t) A+t B) x, x\rangle\langle g((1-t) A+t B) x, x\rangle d t \\
& \leq\langle f(A) x, x)\langle g(A) x, x\rangle \int_{0}^{1}(1-t)^{2} d t+\langle f(B) x, x\rangle\langle g(B) x, x\rangle \int_{0}^{1} t^{2} d t \\
& \quad+(\langle f(A) x, x)\langle g(B) x, x\rangle+\langle f(B) x, x\rangle\langle g(A) x, x\rangle) \int_{0}^{1} t(1-t) d t .
\end{aligned}
$$

It can be easily controlled that

$$
\int_{0}^{1}(1-t)^{2} d t=\int_{0}^{1} t^{2} d t=\frac{1}{3}, \quad \int_{0}^{1} t(1-t) d t=\frac{1}{6} .
$$

When above equalities are taken into account, the proof is complete.

Remark 8 In the inequality (2.10), if we take $x=(1-t) A+t B, a=0$ and $b=1$, we get the inequality (1.5). 
Theorem 9 Let $f, g: I \rightarrow \mathbb{R}$ be an operator convex function on some interval I. Then, for any self-adjoint operators $A$ and $B$ with spectra in $I$, we have the inequality

$$
\begin{aligned}
& \int_{0}^{1}\langle f((1-t) A+t B) x, x\rangle\langle g((1-t) A+t B) x, x\rangle d t \\
& \leq \frac{1}{3 k}(\langle f(A) x, x\rangle\langle g(A) x, x\rangle+\langle f(B) x, x\rangle\langle g(B) x, x\rangle) \\
& \quad+\frac{2}{3 k} \sum_{i=1}^{k-1} f\left\langle\left(\frac{A(k-i)+i B}{k}\right) x, x\right\rangle\left\langle g\left(\frac{A(k-i)+i B}{k}\right) x, x\right\rangle \\
& \quad+\frac{1}{6 k} \sum_{i=0}^{k-1}\left[\left\langle f\left(\frac{A(k-i)+i B}{k}\right) x, x\right\rangle\left\langle g\left(\frac{A(k-i-1)+(i+1) B}{k}\right) x, x\right\rangle\right] \\
& \quad+\frac{1}{6 k} \sum_{i=0}^{k-1}\left[\left\langle f\left(\frac{A(k-i-1)+(i+1) B}{k}\right) x, x\right\rangle\left\langle g\left(\frac{A(k-i)+i B}{k}\right) x, x\right\rangle\right],
\end{aligned}
$$

where $k$ is the number of steps.

Proof The proof is obvious from the proof of Theorem 4 and Theorem 7.

Remark 10 The inequality (2.14) is a general form of the inequality (2.10). When $k=1$ in the inequality (2.14), we get the inequality (2.10).

\section{Competing interests}

The authors declare that they have no competing interests.

\section{Authors' contributions}

All authors contributed equally to the manuscript and read and approved the final manuscript.

\section{Authors' information}

This study is a part of corresponding author's MSc thesis.

\section{Acknowledgements}

This study was supported by The Coordinatorship of Selçuk University's Scientific Research Project (BAP) and The Scientific and Technical Research Council of Turkey (TÜBITAK). The authors wish to express their heartfelt thanks to the referees for their detailed and helpful suggestions for revising the manuscript.

Received: 6 August 2012 Accepted: 9 May 2013 Published: 24 May 2013

\section{References}

1. Pečarić, JE, Proschan, F, Tong, YL: Convex Functions, Partial Orderings and Statistical Applications. Academic Press, New York (1991)

2. Dragomir, SS: Hermite-Hadamard's type inequalities for convex functions of selfadjoint operators in Hilbert spaces. Linear Algebra Appl. 436, 1503-1515 (2012)

3. Dragomir, SS: Hermite-Hadamard's type inequalities for operator convex functions. Appl. Math. Comput. 218, 766-772 (2011)

4. Dragomir, SS: New Hermite-Hadamard-type inequalities for convex functions (II). Comput. Math. Appl. 62, 401-418 (2011)

5. Dragomir, SS: New refinements of the Hermite-Hadamard integral inequality for convex functions and applications. Soochow J. Math. 28(4), 357-374 (2002)

6. Dragomir, SS: Operator Inequalities of the Jensen, Cebysev and Grüss Type. Springer Briefs in Mathematics (2012). doi:10.1007/978-1-4614-1521-3_2

7. Zabandan, G: A new refinement of the Hermite-Hadamard inequality for convex functions. JIPAM. J. Inequal. Pure Appl. Math. 10(2), Article ID 45 (2009)

8. Pachpatte, BG: On some inequalities for convex functions. RGMIA Res. Rep. Coll. 6(E) (2003)

9. Bacak, V, Türkmen, R: New inequalities for operator convex functions. J. Inequal. Appl. 2013, 190 (2013) 
doi:10.1186/1029-242X-2013-262

Cite this article as: Bacak and Türkmen: Refinements of Hermite-Hadamard type inequalities for operator convex functions. Journal of Inequalities and Applications 2013 2013:262

Submit your manuscript to a SpringerOpen ${ }^{\circ}$ journal and benefit from:

- Convenient online submission

- Rigorous peer review

- Immediate publication on acceptance

Open access: articles freely available online

- High visibility within the field

- Retaining the copyright to your article

Submit your next manuscript at $\gg$ springeropen.com 